\title{
Kidney Segmentation in 3D CT Images Using B-Spline Explicit Active Surfaces
}

\author{
Helena R. Torres ${ }^{1,2,}$, Bruno Oliveira ${ }^{1,2, *}$, Sandro Queirós ${ }^{1,2,3}$, Pedro Morais ${ }^{1,3,4}$, Jaime C. Fonseca ${ }^{2}$, Jan D’hooge ${ }^{3}$, \\ Nuno F. Rodrigues ${ }^{1,2,5}$, João L. Vilaça ${ }^{1,5}$ \\ ${ }^{1}$ ICVS/3B'S - PT Government Associate Laboratory, Braga/Guimarães, Portugal. \\ ${ }^{2}$ Algoritmi Center, School of Engineering, University of Minho, Guimarães, Portugal. \\ ${ }^{3}$ Lab on Cardiovascular Imaging and Dynamics, KU Leuven, Belgium. \\ ${ }^{4}$ INEGI, Faculdade de Engenharia, Universidade do Porto, Porto, Portugal. \\ ${ }^{5}$ DIGARC Polytechnic Institute of Cávado and Ave, Barcelos, Portugal.
}

\begin{abstract}
In this manuscript, we propose to adapt the B-Spline Explicit Active Surfaces (BEAS) framework for semi-automatic kidney segmentation in computed tomography (CT) images. To study the best energy functional for kidney CT extraction, three different localized region-based energies were implemented within the BEAS framework, namely localized Chan-Vese, localized Yezzi, and signed localized Yezzi energies. Moreover, a novel gradient-based regularization term is proposed. The method was applied on 18 kidneys from 9 CT datasets, with different image properties. Several energy combinations were contrasted using surface-based comparison against ground truth meshes, assessing their accuracy and robustness against surface initialization. Overall, the hybrid energy functional combining the localized signed Yezzi energy with gradient-based regularization simultaneously showed the highest accuracy and the lowest sensitivity to the initialization. Volumetric analysis demonstrated the feasibility of the method from a clinical point of view, with similar reproducibility to manual observers.
\end{abstract}

Keywords-B-Spline Explicit Active Surfaces, Computed Tomography, Kidney segmentation, Surface analysis, Volumetric analysis.

\section{INTRODUCTION}

$P_{1}$ ERCUTANEOUS renal intervention plays an important role in minimally-invasive kidney interventions (MIKI). MIKI includes percutaneous renal access (PRA) that is a surgical procedure in which a surgical needle is inserted from the abdominal skin surface into a kidney, usually guided by realtime ultrasound (US) imaging, with the aim of providing surgeons with an anatomical target localization [1]. Despite the evolution of medical imaging guidance systems, a precise puncture remains a challenging task for surgeons, often requiring several needle insertion attempts to access the kidney, causing injuries to it or to its surrounding organs [2]. In order to overcome the mentioned issues, the fusion of pre-operative computed tomography (CT) images with intra-operative US have been proposed in others surgical areas, since it offers accurate anatomical information of the target organ and can consequently improve the performance of PRA [3].

The driving problem discussed in this paper is the segmentation of kidney from pre-operative CT images, which allows the extraction of a kidney model to serve as a patientspecific prior shape information for anatomy extraction in interventional US images. Kidney segmentation in CT images can be a challenging task due to the gray scale similarity between the kidney and its neighboring tissues, the presence of the sinus complex in the kidney anatomy, and the image quality.

Over the recent years, several approaches have been proposed for kidney segmentation in CT images, including region growing methods, deformable models, active shape models, level-sets, and registration approaches [4]-[12]. Pohle and Toennies [4], [5] developed an automatic region growing algorithm that estimates the optimized homogeneity criterion from characteristics of the region to be segmented. Despite the improvements over the original approach, their method remains sensitive to the initial seed points. Tsagaan and Shimizu proposed an automatic deformable model-based approach to segment the kidney. Their method is based on a representation of its grey level appearance and its statistical information of the shape, which is incorporated into one energy objective function [6], [7]. In [8], Lin et al. presented an automatic hybrid regionbased and model-based approach for kidney segmentation, which uses an adaptive region growing method to extract kidney within a region of interest (ROI), assuming homogeneity of image intensities. Spiegel et al. [9] introduced an algorithm based on active shape models (ASM) combined with a curvature-based non-rigid registration. This framework learns the kidney mean shape from several training images in order to constrain the segmentation, which links its accuracy to the size of the training data. Khalifa et al. [10] proposed a level-set approach, which combines a probabilistic shape prior and a novel stochastic speed function. This method takes into account spatial interactions and visual appearances along with shape priors. In [11], Cuingnet et al. developed an approach using regression forests to locate the kidney's position and an implicit template model to refine its segmentation, driven by a kidney probability map. More recently, an automatic method based on multi-atlas image registration was proposed by Yang et al. [12].

Recently, Barbosa et al. proposed the B-spline Explicit Active Surfaces (BEAS) framework for real-time segmentation of heterogeneous datasets. The BEAS framework emerged from the concept of active contours, first introduced in [14]. In active contours, an evolving interface is propagated to recover the boundary of an object through the minimization of a given energy functional. Duan et al. introduced a computationally

\footnotetext{
$\overline{* \text { First joint authorship. }}$
} 
efficiency explicit interface representation through the introduction of the active geometric functions (AGF) [15]. The BEAS method extends the concept of AGF to a B-spline formulation, as originally proposed in [16]. By allowing the surface evolution according to localized region-based energies, an accurate and fast segmentation in heterogeneous images is achieved.

In this paper, we propose to adapt BEAS for fast semiautomatic kidney segmentation in 3D CT images. In this sense, and in order to study the best energy functional for kidney CT extraction, three different localized region-based energies are presented and compared, namely localized Chan-Vese (LCV), localized Yezzi (LY), and signed localized Yezzi (SLY) energies. Moreover, a novel gradient-based regularization term is proposed, adding edge-based features into the surface evolution process and increasing the methods' capture range (i.e. its robustness against the initialization).

The rest of this paper is organized as follows. In section II, the general formulation of the BEAS framework and proposed energy functional are presented. In section III, the experiments are outlined, including implementation details, data description and ground truth construction. In section IV, the performance of the method is assessed for different energy combinations, through quantitative results on real CT images. In section V, the results are discussed, with the main conclusions being given in section VI.

\section{METHODOLOGY}

In this section, a brief description of the BEAS framework is presented (section II-A). Moreover, the proposed energy functional for kidney segmentation is described (section II-B), with the different studied localized region-based energies and proposed regularization term (section II-C).

\section{A. B-Spline Explicit Active Surfaces}

The fundamental concept of the BEAS framework is to define the boundary of an object as an explicit function. Geometrically, this implies that one of the coordinates of the points on a surface is given explicitly as a function of the remaining coordinates. Such explicit relation can be mathematically defined as:

$$
\psi: \mathbb{R}^{n-1} \mapsto \mathbb{R}, x_{1}=\psi\left(\boldsymbol{x}^{*}\right)
$$

where $\boldsymbol{x}$ is a point of coordinates $\left\{x_{1}, \ldots, x_{n}\right\}$ and $\boldsymbol{x}^{*}=\left\{x_{2}, \ldots, x_{n}\right\}$. Note that the explicit form of the interface limits the topology of the contour. Nevertheless, this formulation fits well to the kidney boundary, owing its star-like and smoothed shape.

Bernard et al. proposed a formulation where the interface function is modeled as a continuous parametric function expressed on a B-spline basis [16]. Inspired by their work, the BEAS method expresses $\psi$ as a linear combination of B-spline basis functions:

$$
x_{1}=\psi\left(x_{2}, \ldots, x_{n}\right)=\sum_{\mathrm{k} \in \mathbb{Z}^{\mathrm{n}-1}} \mathrm{c}[\mathbf{k}] \beta^{\mathrm{d}}\left(\frac{\mathbf{x}^{*}}{\mathrm{~h}}-\mathbf{k}\right),
$$

where $\beta^{\mathrm{d}}($.$) is the uniform symmetric (n-1)$-dimensional $\mathrm{B}$-spline of degree $d$. The knots of the B-splines are located on a rectangular grid defined on the chosen coordinate system, with a regular spacing given by $h$. The coefficients of the B-spline representation are gathered in $c[\boldsymbol{k}]$ and the minimization of an energy functional can be directly obtained in terms of these coefficients, as derived in [13].

\section{B. Proposed Energy Functional}

To evolve the surface, it is crucial to define an appropriate energy criterion. This energy functional can be expressed in terms of two categories, which include edge-based terms or region-based terms. In edge-based active contours, image gradients are used to identify object boundaries [17]. The main issue with these terms is the sensibility to the image noise. Chan and Vese proposed a region-based active contour to overcome the limitations of edge-based approaches [18]. Their approach models the foreground and background regions statistically in a global way, performing the partition of the image into homogeneous regions. Despite its advantages in comparison with edge-based active contours, the global region-based approach is not ideal for segmenting heterogeneous objects. Lankton et al. [19] introduced the concept of localized regionbased energies to improve the abovementioned methodology. In the segmentation process, the evolving interface is propagated taking into account the properties of the local regions around a given point of the interface. This approach makes the segmentation process robust to heterogeneous images.

In this sense, a localized region-based energy functional can be defined in terms of a generic force function $F$ as:

$$
E=\int_{\Omega} \delta_{\phi}(\boldsymbol{x}) \int_{\Omega} B(\boldsymbol{x}, \boldsymbol{y}) \cdot F(\boldsymbol{y}) d \boldsymbol{y} d \boldsymbol{x},
$$

where

$$
F(\boldsymbol{y})=f_{\text {in }}(\boldsymbol{y}) \cdot H_{\phi}(\boldsymbol{y})+f_{\text {out }}(\boldsymbol{y}) \cdot\left(1-H_{\phi}(\boldsymbol{y})\right)
$$

and, $f_{\text {in }}$ and $f_{\text {out }}$ provide energy criteria for the interior and the exterior region of the interface $\Gamma . \delta_{\phi}(\boldsymbol{x})$ specifies the interface and $H_{\phi}(\boldsymbol{x})$ is the Heaviside function that specifies the interior of the contour. The exterior of the contour is defined as $\left(1-H_{\phi}(\boldsymbol{x})\right) . B(\boldsymbol{x}, \boldsymbol{y})$ is a mask function in which the local parameters are estimated to drive the contour evolution. The mask $B$ around point $\boldsymbol{x}$ is defined as the set of points belonging to the normal direction of $\boldsymbol{x}$ and whose distance is lower than a parameter $\rho$ [13], thus defined as:

$$
B(\boldsymbol{x}, \boldsymbol{y})=\left\{\begin{array}{c}
1, \text { if } \boldsymbol{y}=\boldsymbol{x}+k \times \widehat{\boldsymbol{N}}, k \in[-\rho, \rho] \\
0, \text { otherwise }
\end{array}\right.
$$

where $\widehat{\boldsymbol{N}}$ is the normal vector to the interface at point $\boldsymbol{x}$. This definition of $B$ allows the reduction of the computational time in comparison with the cubic mask originally proposed by Lankton et al. [19]. 
In order to minimize the abovementioned energy, a gradient descent optimization is used. In the BEAS framework, the energy can be directly minimized with respect to the B-spline coefficients $c[\mathbf{k}]$, given by the following evolution equations:

$$
\begin{gathered}
c[\boldsymbol{k}]^{(t+1)}=c[\boldsymbol{k}]^{(t)}-\lambda_{\text {step }} \frac{\partial E}{\partial c[\boldsymbol{k}]^{\prime}} \\
\frac{\partial E}{\partial c\left[\boldsymbol{k}_{\boldsymbol{i}}\right]}=\int_{\Gamma}\left(\bar{g}_{L R}\left(\boldsymbol{x}^{*}\right)+\bar{g}_{E d g e}\left(\boldsymbol{x}^{*}\right)\right) \beta^{d}\left(\frac{\boldsymbol{x}^{*}}{h}-\boldsymbol{k}_{\boldsymbol{i}}\right) d \boldsymbol{x}^{*},
\end{gathered}
$$

where $t$ is the current iteration number and $\lambda_{\text {step }}$ is a parameter controlling the step at each iteration, as originally proposed in [13]. $\bar{g}_{L R}\left(\boldsymbol{x}^{*}\right)$ and $\bar{g}_{E d g e}\left(\boldsymbol{x}^{*}\right)$ are functions that reflect the features of the object to be segmented.

\section{Localized energy functionals}

Hereto, three localized region-based energies that can be used to extract the kidney from CT images are presented, together with a novel gradient-based regularization term. The performance of each method will be addressed in section IV.

\section{1) Localized Chan-Vese (LCV) Energy}

The energy proposed by Chan and Vese is based on the Mumford-Shah method and the correspondent function $F$ is given as:

$$
\begin{aligned}
F(\boldsymbol{y})=H_{\phi}(\boldsymbol{y}) \cdot\left(I(\boldsymbol{y})-u_{x}\right)^{2} & \\
& +\left(I(\boldsymbol{y})-v_{x}\right)^{2} \cdot\left(1-H_{\phi}(\boldsymbol{y})\right),
\end{aligned}
$$

where $u_{x}$ and $v_{x}$ are the mean intensities inside and outside of the evolving interface at point $\boldsymbol{x}$, calculated using mask $B$.

The LCV energy aims to partition the image into regions, modeling the inside and outside of the contour as a constant intensity that is given by the average intensity in the respective side of the contour. In other words, the pixels in each region are penalized according to the intensity variation with respect to the corresponding region's average intensity. The energy minimization is achieved using the feature function given by:

$$
\bar{g}_{L R}\left(\boldsymbol{x}^{*}\right)=\left(\bar{I}\left(\boldsymbol{x}^{*}\right)-u_{x}\right)^{2}-\left(\bar{I}\left(\boldsymbol{x}^{*}\right)-v_{x}\right)^{2} .
$$

For clarity's sake, considering a generic function $h(x)$ in $\mathbb{R}^{n}$, $\bar{h}$ is noted as the restriction of $h$ over the interface $\Gamma$ in $\mathbb{R}^{n-1}$. Thus, $\overline{\mathrm{I}}\left(\boldsymbol{x}^{*}\right)$ corresponds to the image value at position $\boldsymbol{x}=\left\{x_{1}=\psi\left(\boldsymbol{x}^{*}\right), x_{2}, \ldots, x_{n}\right\}$.

\section{2) Localized Yezzi (LY) Energy}

Yezzi et al. [20] proposed an energy functional that guarantees that the statistics of the image, such as the average intensity of each region, evolves in order to have the maximum separation between them. Therefore, the energy relies on the assumption that the interior and the exterior of the contour have the largest difference in average intensities. With this in mind, when compared to the LCV energy, this functional models the foreground and background as having maximally separated intensities, instead of locally homogeneous only.

Equations (10) and (11) present the $F$ function and respective feature function.

$$
\begin{gathered}
F(\boldsymbol{y})=\left(u_{x}-v_{x}\right)^{2} \\
\bar{g}_{L R}\left(\boldsymbol{x}^{*}\right)=\frac{\left(\bar{I}\left(\boldsymbol{x}^{*}\right)-u_{x}\right)^{2}}{A_{u}}-\frac{\left(\bar{I}\left(\boldsymbol{x}^{*}\right)-v_{x}\right)^{2}}{A_{v}} .
\end{gathered}
$$

where $A_{u}$ and $A_{v}$ represent the areas inside and outside of the contour, respectively.

\section{3) Signed Localized Yezzi (SLY) Energy}

The Yezzi energy previously referred does not specify the intensity relation between foreground and background. In other words, this energy does not take into account if, e.g., the foreground region has to be brighter or darker than the background. To explicitly specify the expected relation between both regions, Queirós et al. [21] proposed a signed version of the Yezzi energy, expressed in terms of $F$ as:

$$
F(\boldsymbol{y})=u_{x}-v_{x}
$$

According to eq. (11), the contour is forced to evolve so that the interior of the interface has higher intensities than its exterior. Such functional assumes that the object to be segmented is brighter than the background. However, this functional can be easily modified to segment dark objects, switching $u_{x}$ and $v_{x}$ in the equation. The feature function for the evolution in respect to eq. (11) is given as:

$$
\bar{g}_{L R}\left(\boldsymbol{x}^{*}\right)=\left(\bar{I}\left(\boldsymbol{x}^{*}\right)-u_{x}\right)+\left(\bar{I}\left(\boldsymbol{x}^{*}\right)-v_{x}\right) .
$$

In Fig.1B is shown that the SLY energy forces the evolving interface to move towards the kidney boundary where the intensities inside the contour in the mask will be higher than the intensities of the outside.

\section{4) Gradient-based regularization term (GRT)}

Since well-defined boundaries are expected in CT images, we proposed an edge-based regularization term that drives the segmentation for boundary positions, making the strategy less dependent of the initialization and local minima. In the present implementation, we estimate the optimal edge direction using the derivative of the surface's normal profiles (computed using mask $B$ ). Note that the intensity profiles are smoothed by a 1-D Gaussian kernel prior to the derivative. Moreover, since the kidney intensity is brighter than its neighborhood, only brightto-dark transitions are relevant in the abovementioned edge direction estimation technique. Thus, the edge position is defined as the profile point with the minimum derivative value, $\boldsymbol{y}_{\text {min }}^{*}$, therefore defining our proposed gradient-based regularization as:

$$
\bar{g}_{E d g e}\left(\boldsymbol{x}^{*}\right)= \begin{cases}1 & \text { if } \boldsymbol{y}_{\min }^{*}>\boldsymbol{x}^{*} \\ -1 & \text { if } \boldsymbol{y}_{\min }^{*}<\boldsymbol{x}^{*}\end{cases}
$$

In Fig. 1C, an example derivative profile is shown. One can observe that a surface point will evolve towards the kidney boundary, where the derivative profile value is minimum. 

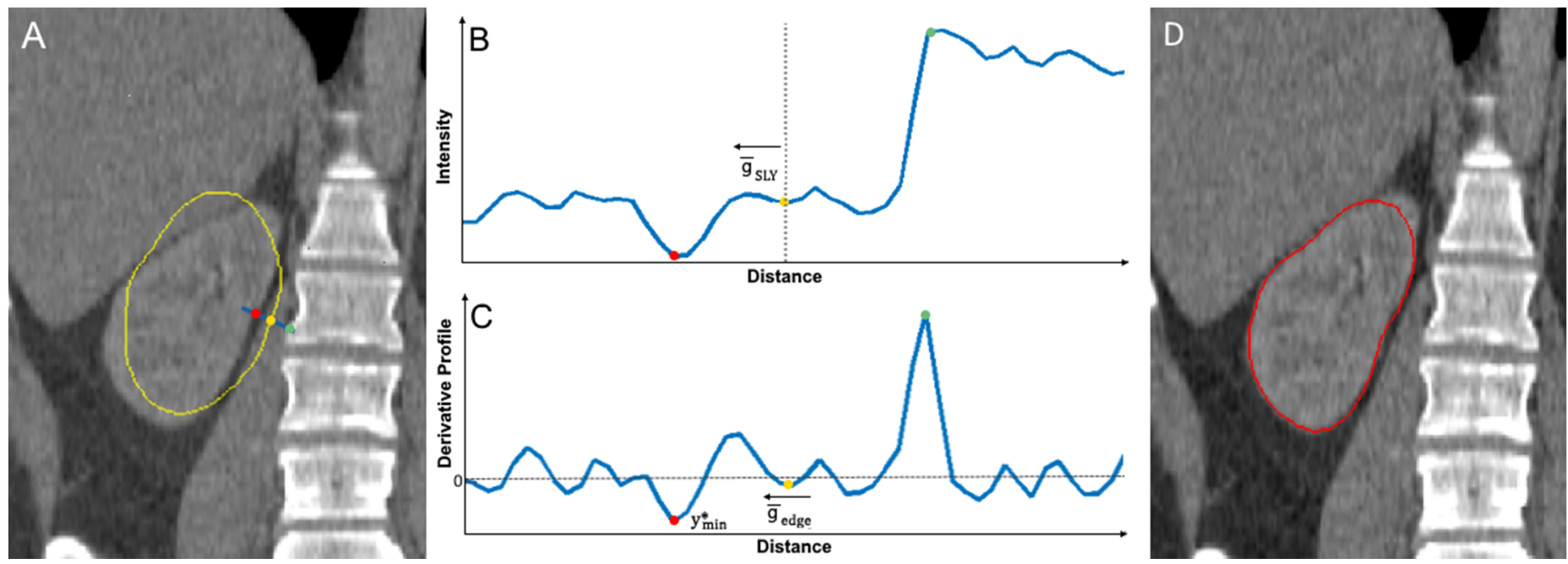

Fig. 1. (A) Example of CT image and the evolving interface (yellow line); (B) Image intensity profile extracted for a given surface point (yellow dot); (C) Derivative profile of (B); (D) Result of semi-automatic segmentation. Note that the red, yellow and green dot in (A), (B), and (C) represent the transition kidney-background, the evolving interface and the transition background-spine, respectively.

\section{EXPERIMENTS}

\section{A. Dataset}

The performance of the proposed semi-automatic segmentation method was evaluated on a database of $9 \mathrm{CT}$ images with different properties. In each dataset, both left and right kidneys were individually assessed.

The parameters used during acquisition of the CT images were set to $120.0 \mathrm{kV}$ and between 265.0 and $445 \mathrm{~mA}$. The pixel spacing ranged from 0.61 to $0.96 \mathrm{~mm}$ and the spacing between slices ranged from 1.5 to $3 \mathrm{~mm}$. The number of slices was between 502 and 797. Each axial slice had a spatial resolution of $512 \times 512$ pixels.

\section{B. Ground Truth}

The kidney surface's ground truth was manually constructed by one observer. The manual segmentation was performed twice (henceforward referred as Manual1 and Manual2) in the Medical Imaging Interaction Toolkit (MITK) software [22]. The two ground truths for each kidney were compared with the results of the semi-automatic framework and with each other in order to assess accuracy and reproducibility of both automated and manual delineations.

\section{Initialization of the Contour}

The first step of active contour-based segmentation strategies is the selection of an initial contour that will be evolved to the desired boundary. To initialize the BEAS method, a non-expert user (blind to the delineations performed by the first observer during ground truth generation) drew fourteen points in the kidney and an ellipsoid was fitted to this data. The fitted ellipsoid was used as initialization during semi-automatic segmentation with BEAS.

In order to study the robustness of each energy functional against the initialization used, three different initializations (with progressive difficulty) were gathered for each kidney, and will be used in section IV.

\section{Implementation details}

In the BEAS framework, the definition of certain parameters is essential. Firstly, an appropriate coordinate system according to the topology of the object to be segmented needs to be defined. Due to the ellipsoid shape of the kidney, a spherical coordinate system was chosen. Thus, the radial coordinate of the points within the surface will be given as a function of the azimuth and zenith angles. Note that the principal axis of the initialization ellipsoid was used as the principal axis of the BEAS spherical space.

Secondly, the B-spline scale, the number of surface points and the size of mask $B$ needs to be defined. It is important to notice that the choice of the number of surface points used is explicitly linked with the choice of the B-spline scale, therefore being paramount to define an ideal relation between these two parameters. In one hand, if a low number of points is used, details of the real boundary of the kidney may be lost. On the other hand, a high number of points increases the computational burden of the method and may result in a jagged (i.e. nonsmooth) contour. The B-spline scale is related with the computation of the B-spline coefficients, which implicitly control the surface smoothing. To avoid over-smoothing, the B-spline scale has to be low. In the current experiments, the B-spline scale was empirically set to $2^{2}$ and 32 points were used to define each direction on the rectangular grid of the spherical domain. Regarding mask $B$, the radius of the normal vector was empirically set to $15 \mathrm{~mm}$.

This framework was implemented in MATLAB and the feasibility of the adopted method was assessed through two different analyses, namely surface and volumetric based comparisons.

\section{RESUlts}

\section{A. Surface Analysis}

Table I summarizes the performance for different energy combinations, assessed in terms of average point-to-surface 
distance (P2S) between the semi-automatic segmentation and the ground truth surface for the 18 segmented kidneys. To assess robustness against initialization, the results presented are referred to three different initial surfaces. In the first situation (INIT1), the initial fitted ellipsoid was drawn so that the 95 percentile of the P2S errors (PRC95) against manual segmentation were below $7.5 \mathrm{~mm}$. For the second initialization (INIT2), the 95 percentile was between $7.5 \mathrm{~mm}$ and $12.5 \mathrm{~mm}$. In its turn, the third initialization (INIT3) was drawn so that the initial surface is farther away from the true kidney boundaries, with a 95 percentile P2S error above $12.5 \mathrm{~mm}$ (near $15 \mathrm{~mm}$, in average). The average $\mathrm{P} 2 \mathrm{~S}$ of the initializations with respect to manual segmentation is also presented in Table I.

Table II presents a broader surface error analysis for the energy functional showing the best performance in terms of average P2S errors (SLY + GRT, Table I). This surface analysis includes the Dice coefficient (that compares the overlap between two surfaces), the 95 percentile distance (PRC95, defined as the 95 percentile of $\mathrm{P} 2 \mathrm{~S}$ errors), and the percentage of "good contours" (GC, that accounts for the percentage of cases for which the average P2S error is lower than $5 \mathrm{~mm}$ ). Moreover, Table II presents the respective distance metrics between two intra-observer manual delineations.

To visually demonstrate the method's performance, Fig. 2 gives two example results for the three initializations used.

\section{B. Volumetric Analysis}

The comparison of the volumes of the segmented kidney surface and the manual one can be done through a volumetric analysis. This analysis can be seen in Fig. 3, which presents the Bland-Altman plots [23] for each initialization using the first manual segmentation as reference. In these analyses, the biases (average difference between methods) and limits of agreement (LOA, 1.96 $\sigma$ ) were assessed. The volumetric comparison was also performed between both manual delineations.

\section{Computational Analysis}

As referred, one of the great advantages of the BEAS method is the reduced computational burden. Table III presents the average computational time for each segmentation energy and for each initialization. Furthermore, Table III also presents the average number of iterations needed by the algorithm to convergence. Note that a $3.6 \mathrm{GHz}$ Core computer with $\mathrm{CPU}$ i7-4740 and 16 GB of RAM was used.

\section{DISCUSSION}

Table I shows the performance of the BEAS method applied to kidney CT segmentation. Firstly, it is important to notice that the P2S errors are lower for all cases when starting from INIT1 $(P C R 95<7.5 \mathrm{~mm})$ and higher when using INIT3 (PCR95 > $12.5 \mathrm{~mm})$, as expected for active contour and level-set based approaches [14],[19]. In this way, it is possible to verify the performance of the segmentation method for close and far initializations.

Analyzing the results for each energy combination, it is possible to observe that different energies result in different values of average P2S errors. Regarding the LCV energy, a
TABLE I.

KIDNEY SEGMENTATION PERFORMANCE IN TERMS OF P2S (MM) FOR DIFFERENT ENERGY COMBINATIONS AND INITIALIZATIONS

\begin{tabular}{cccc}
\hline \hline & INIT1 & INIT2 & INIT3 \\
\hline Initialization & $2.90 \pm 0.55$ & $4.41 \pm 0.74$ & $5.56 \pm 0.70$ \\
LCV & $2.15 \pm 0.53$ & $3.43 \pm 0.94$ & $4.99 \pm 0.78$ \\
LCV + GRT & $1.66 \pm 0.42$ & $2.80 \pm 0.80$ & $4.35 \pm 0.68$ \\
LY & $2.70 \pm 2.18$ & $3.03 \pm 1.77$ & $4.44 \pm 2.25$ \\
LY + GRT & $1.50 \pm 0.43$ & $1.82 \pm 0.67$ & $2.47 \pm 1.46$ \\
SLY & $2.02 \pm 0.68$ & $2.52 \pm 1.13$ & $3.92 \pm 1.72$ \\
SLY+ GRT & $1.48 \pm 0.44$ & $1.66 \pm 0.52$ & $2.52 \pm 1.97$ \\
\hline \hline
\end{tabular}

TABLE II.

KIDNEY SEGMENTATION PERFORMANCE USING SIGNED LOCALIZED YEZZI ENERGY WITH GRADIENT-BASED REGULARIZATION TERM

\begin{tabular}{|c|c|c|c|c|c|}
\hline & & $\begin{array}{c}\text { Dice } \\
(\%)\end{array}$ & $\begin{array}{l}\text { P2S } \\
(\mathrm{mm})\end{array}$ & $\begin{array}{c}\text { PRC95 } \\
(\mathrm{mm})\end{array}$ & $\begin{array}{l}\text { GD } \\
(\%)\end{array}$ \\
\hline \multirow{3}{*}{ 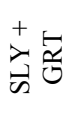 } & INIT1 & $92.6 \pm 2.1$ & $1.48 \pm 0.44$ & $4.39 \pm 2.11$ & 100 \\
\hline & INIT2 & $91.5 \pm 3.2$ & $1.66 \pm 0.52$ & $5.05 \pm 2.18$ & 100 \\
\hline & INIT3 & $88.8 \pm 7.5$ & $2.52 \pm 1.97$ & $8.71 \pm 6.64$ & 94,4 \\
\hline \multicolumn{2}{|c|}{ Manual1 vs Manual2 } & $94.6 \pm 0.9$ & $1.06 \pm 0.19$ & $2.85 \pm 0.51$ & 100 \\
\hline
\end{tabular}

direct relation was also found between the accuracy of the segmentation result and the quality of the initialization. Moreover, this energy typically presented the worst result for the different scenarios when compared with the remaining energies. This result can be explained by the energy function used, which relies on the identification of two homogeneous regions. As such, wrong initializations make the method sensitive to local homogenous regions and, therefore, to local minima.

When using the LY energy, despite some very good results in a few CT images, this energy typically produces less accurate segmentations. In abdominal CT images, the kidney may be too close to the spine that has higher intensities than all other tissues. In the LY energy, the segmentation evolution tries to maximize the difference between the intensities in the inside and outside of the contour. The fact that the difference between the spine and its surrounding tissues is higher than the difference between the kidney and its neighbors explains the less accurate segmentation in some images.

The SLY energy presented more accurate results for all initializations. In the current work, the SLY was implemented so that the interior of the surface is brighter than its exterior. Note that the kidney has higher intensities than the surrounding tissues. Such explicit prior allows for an increased accuracy and robustness against different appearances of the abdominal region in the $\mathrm{CT}$ images used. In comparison with the LCV energy, the probability of finding local minima is lower owing to the fact that this functional tries to find a region where the inside and outside have maximum separated average intensities, which probably corresponds to regions near tissues' boundaries. Comparing with the LY energy, the definition of the specific relation between the interior and exterior intensities allows to specify the correct direction for surface evolution. As the spine corresponds to a large dark-to-bright transition, the SLY energy 


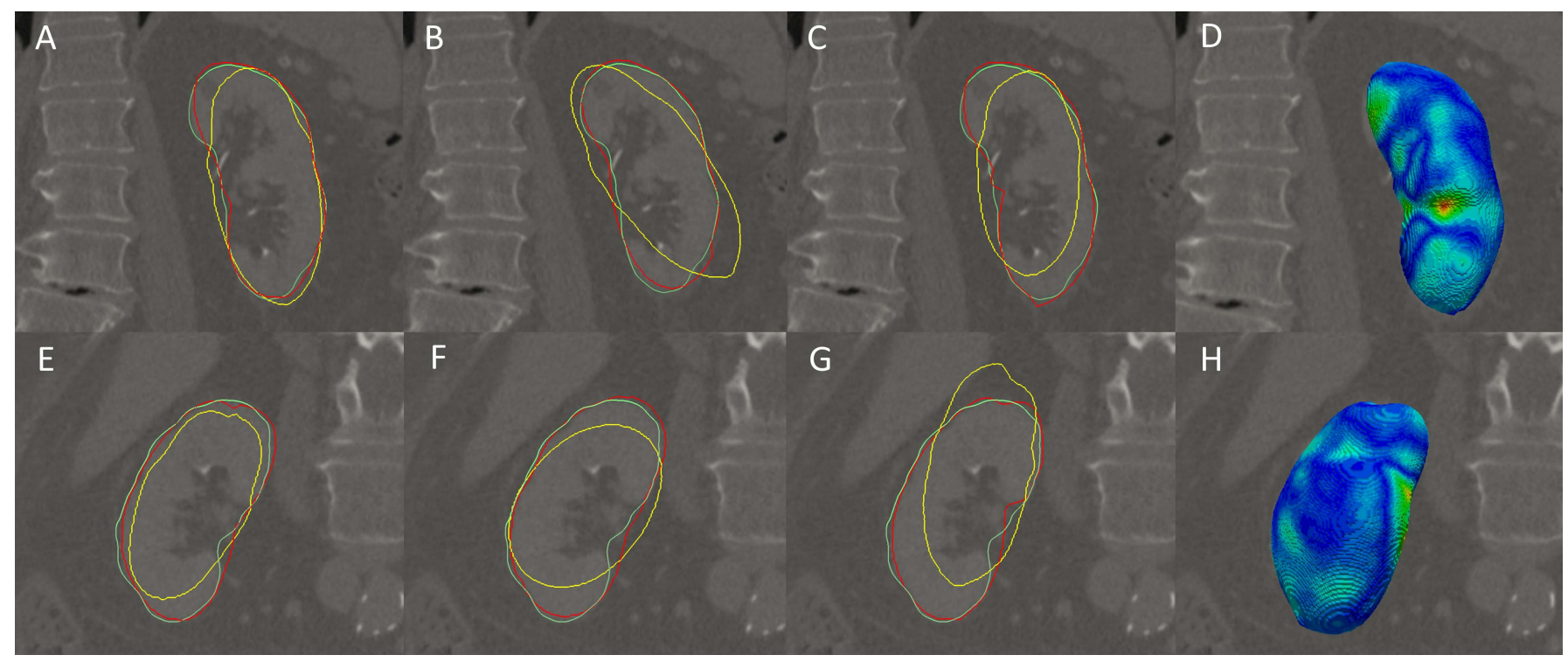

Fig. 2. Segmentation results for two example kidneys. (A), (E) results for INIT1; (B), (F) results for INIT2; (C), (G) results for INIT3 (yellow: initialization; red: semi-automatic segmentation; green: ground truth of Manual1). (D) and (H) present the semi-automatically segmented 3D surface (for INIT1), overlaid with the P2S errors.
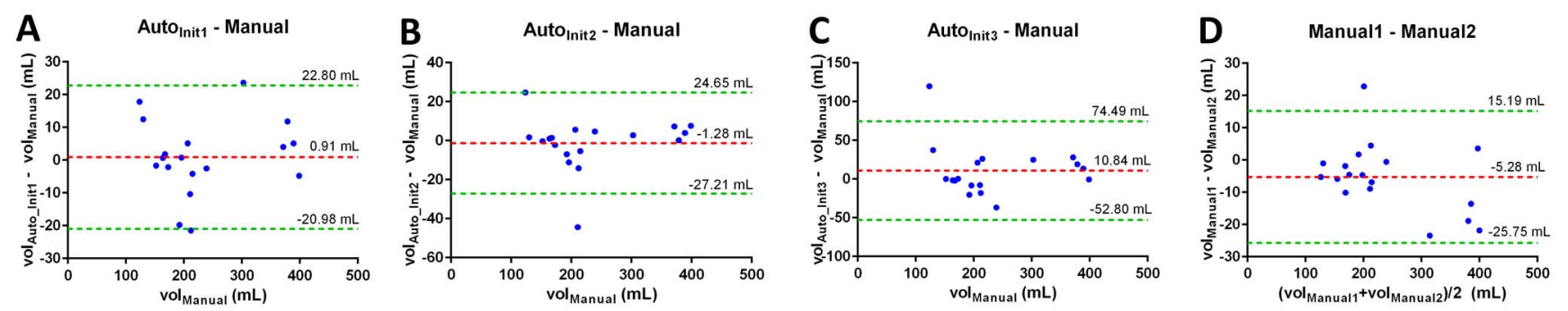

Fig. 3. Bland-Altman analysis for (A) INIT1; (B) INIT2; (C) INIT3; and (D) both manual delineations.

evolves away from the spine boundaries (see Fig. 1B). Overall, this energy functional is more robust and accurate (Table I).

The addiction of the proposed regularization term allowed to decrease the errors between the semi-automatic and manual segmentations (Table I). This superior performance is due to the clear definition of the kidney boundaries in CT images, which force the surface to evolve towards positions with higher image gradients (Fig. 1C). Note that the edge-based term is crucial to quickly push the contour when far from the true boundaries, therefore increasing the capture range of the proposed method. When closer to the boundary, the result is mainly refined through the localized region-based energy. Moreover, since the GRT searches for specific transitions (bright-to-dark), the presence of surrounding interfaces is penalized, making the method more robust against worst initializations.

The analysis of Table I allow us to conclude that the most accurate segmentation was obtained with the SLY energy combined with the GRT term. Table II presents an extended analysis of the segmentation results with this energy functional. Regarding the average Dice coefficient, differences were found between the automatic segmentation for each initialization, in respect to Manual1, and the two manual segmentations. Nevertheless, the automated segmentation and manual segmentation 1 showed a good percentage of similarity. The errors of the segmentation can be seen in the distance metrics (P2S and PRC95). The difference between the results for the automatic segmentation and the two manual segmentations in terms of P2S and PRC95 corroborates the importance of a correct initialization. Note that the P2S errors increase with the distance of the initialization. Nonetheless, the differences between INIT1 and INIT2 results are not significant, which does not occur between INIT2 and INIT3. For this reason, one can conclude that the method obtains very accurate segmentations for initializations with up to $12.5 \mathrm{~mm}$ of initial errors (INIT1 and INIT2), but has slightly higher errors for worst initializations (with initial errors above $12.5 \mathrm{~mm}$, INIT3). Lastly, regarding GC, the average error for all images is below $5 \mathrm{~mm}$ for INIT1 and INIT2. For INIT3, only one in eighteen kidney segmentations presented an average error above $5 \mathrm{~mm}$.

In Fig. 2 it is possible to visually verify that the SLY energy with GRT results in segmentations close to the observer for the three initializations, which proves the robustness of the method. Note that the highest errors occur in the hilum of the kidney (Fig. 2D and Fig.2H).

When assessing the agreement between manually and semi-automatically computed volumes (Fig. 3), no statistically significant biases (red dashed line) were observed for the different initializations $(p>0.05$ in a two-tailed paired T-test 
TABLE III.

AVERAGE TIMES (S) OF SEGMENTATION AND NUMBER OF ITERATIONS

\begin{tabular}{ccccccc}
\hline \hline & \multicolumn{2}{c}{ INIT1 } & \multicolumn{2}{c}{ INIT2 } & \multicolumn{2}{c}{ INIT3 } \\
\hline & Time & Its & Time & Its & Time & Its \\
\hline LCV & 1.1 & 41.4 & 0.8 & 33.1 & 0.8 & 33.7 \\
LCV + GRT & 1.3 & 51.9 & 0.9 & 34.5 & 0.7 & 28.5 \\
LY & 2.1 & 96.1 & 2.4 & 101.2 & 2.8 & 118.2 \\
LY + GRT & 1.5 & 57.3 & 1.7 & 68.2 & 2.3 & 88.3 \\
SLY & 1.4 & 61.3 & 1.9 & 80.8 & 2.3 & 98.3 \\
SLY+ GRT & 1.6 & 65.2 & 1.6 & 64.3 & 2.4 & 93.1 \\
\hline
\end{tabular}

against 0). Moreover, narrow LOAs (green dashed lines) were obtained, particularly for INIT1. In fact, for INIT1 and INIT2, the LOAs proved to be statistically similar to the intra-observer ones $(p>0.05$ in a two-tailed F-Test against the intra-observer LOA), which suggests the equivalence of semi-automatic and manual volume measurements. Only for worst initializations (INIT3), a broader LOA was obtained $(p<0.05)$.

Table III reports the average computational time for the segmentation. A fast segmentation was obtained for the experiments (average time lower than $2.8 \mathrm{~s}$ ), proving the clear advantages of the BEAS framework for fast 3D segmentation problems and, therefore, showing the potential of this framework for future real-time image-fusion frameworks for MIKI procedures.

\section{CONCLUSION}

In this paper, the BEAS framework was adapted for kidney CT segmentation. From the different localized region-based energies studied, with and without the proposed gradient-based regularization term, the best results were obtained with the SLY energy coupled with our gradient-based term. The proposed algorithm shown accurate segmentation results, with reduced sensitivity to the initialization provided. Moreover, the method proved to be computationally efficient, taking in average less than $2 \mathrm{~s}$ to segment a kidney. In summary, the proposed segmentation method proved to be successful and feasible for pre-operative CT segmentation.

In future work, we intend to develop an automatic kidney detection algorithm to fully automatize the segmentation and, thus, obtain a user-independent methodology. Moreover, we intend to extend the proposed segmentation methodology for intra-operative ultrasound datasets.

\section{ACKNOWLEDGEMENT}

The authors acknowledge FCT - Fundação para a Ciência e a Tecnologia, Portugal, and the European Social Found, European Union, for funding support through the Programa Operacional Capital Humano (POCH) in the scope of the $\mathrm{PhD}$ grants SFRH/BD/93443/2013 (S. Queirós) and SFRH/BD/95438/2013 (P. Morais).

\section{REFERENCE}

[1] P.L. Rodrigues, N.F. Rodrigues, J. Fonseca, E. Lima, and J.L. Vilaça, "Kidney Targenting and Puncturing during Percutaneous Nephrolithotomy: Recent Advances and Future Perspectives," Journal of Endourology, vol 27, pp.826834, 2013.
[2] J. d. 1. Rosette, D. Assimos, M. Desai, J. Gutierrez, J. Lingeman, R. Scarpa, et al," "The clinical research office of the endourological society percutaneous nephrolithotomy, and outcomes in 5803 patients," Journal of endourology, vol. 25, pp.1117, 2011.

[3] W.G. Wein, S.Brunke, A.Khamene, M.R. Callstrom, and N. Navab, "Automatic CT-ultrasound registration for diagnostic imaging and image guided intervention," Medical Image Analysis, vol. 12, pp. 577-585, Oct 2008. [4] R. Pohle and K. D. Toennies, "A new approach for model-based adaptive region growing in medical image analysis," in Computer Analysis of Images and Patterns, vol. 2124, pp.238-246, 2001.

[5] R. Pohle and K. D. Toennies, "Segmentation of medical images using adaptive region growing," in Proc. Int. Soc. Opt. Eng. (SPIE), vol. 4322, pp. 1337-1346, 2001 .

[6] B. Tsagaan, A. Shimizu, H. Kobatake, K. Miyakawa, and Y. Hanzawa, "Segmentation of kidney by using a deformable model," in Int. Conf. Image Processing, vol. 3, pp. 1059-1062, 2001.

[7] B. Tsagaan,A. Shimizu,H. Kobatake, andK. Miyakawa, "An automated segmentation method of kidney using statistical information," in Proc. Medical Image Computing and Computer Assisted Intervention, vol. 1, pp. 556-563, 2002.

[8] D. Lin, C. Lei, and S. Hung, "Computer-aided kidney segmentation on abdominal CT images,” IEEE Trans. Inf. Technol. Biomed., vol. 10, no. 1, pp. 59-65, Jan. 2006.

[9] Spiegel M., Hahn D. A., Daum V., Wasza J., Hornegger, J. "Segmentation of kidneys using a new active shape model generation technique based on nonrigid image registration," Comput. Med. Imag. Grap., vol. 33, no.1, pp.29-39, 2009.

[10] Khalifa F., Elnakib A., Beache G. M., et al. “3D kidney segmentation from CT images using a level set approach guided by a novel stochastic speed function," in Proc.MICCAI 2011. Springer Berlin Heidelberg, pp.587-594, 2011

[11] Cuingnet R., Prevost R., Lesage D., et al. "Automatic detection and segmentation of kidneys in 3D CT images using random forests", in Proc. MICCAI 2012. Springer Berlin Heidelberg, pp. 66-74, 2012.

[12] G. Yang, J. Gu, Y. Chen, W. Liu, L. Tang, H. Shu, and C. Toumoulin, "Automatic Kidney Segmentation in CT Images based on Multi-atlas Image Registration," in 36th Annual International Conference of the IEEE Engineering in Medicince and Biology Society, 2014, no. Mi, pp. 5538-5541.

[13] D. Barbosa, T. Dietenbeck, J. Schaerer, J. D'hooge, D. Friboulet, and O. Bernard, "B-Spline Explicit Active Surfaces: An Efficient Framework for Real-Time 3-D Region-Based Segmentation," IEEE Trans. Image Process., vol. 21, no. 1, pp. 241-251, 2012.

[14] M. Kass, A. Witkin, and D. Terzopoulos, "Snakes: Active contour models," Int. J. Comput. Vis., vol. 1, no. 4, pp. 321-331, Jan. 1988.

[15] Q. Duan, E. D. Angelini, and A. F. Laine, "Real-time segmentation by active geometric functions," Comput. Methods Programs Biomed., vol.98, no. 3, pp. 223-230, Jun. 2010.

[16] O. Bernard, D. Friboulet, P. Th'evenaz, and M. Unser, "Variational BSpline level-set: A linear filtering approach for fast deformable model evolution," IEEE Transactions on Image Processing, vol. 18, pp. 1179-1191, 2009.

[17] V. Caselles, R. Kimmel, and G. Sapiro, "Geodesic active contours," Int. J. Comput. Vis., vol. 22, no. 1, pp. 61-79, Feb. 1997.

[18] T. Chan and L. Vese, "Active contours without edges," IEEE Trans. Image Process., vol. 10, no. 2, pp. 266-277, Feb. 2001.

[19] S. Lankton and A. Tannenbaum, "Localizing region-based active contours," IEEE Trans. Image Process., vol. 17, no. 11, pp. 2029-2039, Nov. 2008.

[20] J. A. Yezzi, A. Tsai, and A.Willsky, "A fully global approach to image segmentation via coupled curve evolution equations," J. Vis. Comm. Image Rep., vol. 13, no. 1, pp. 195-216, Mar. 2002.

[21] S. Queirós, D. Barbosa, B. Heyde, P. Morais, J. L. Vilaça, D. Friboulet, O. Bernard, and J. D'hooge, "Fast automatic myocardial segmentation in 4D cine CMR datasets," Med. Image Anal., vol. 18, no. 7, pp. 1115-1131, 2014. [22] I. Wolf, M. Vetter, I. Wegner, M. Nolden, T. Bottger, M. Hastenteufel, M. Schobinger, T. Kunert, and H.-P. Meinzer, "The Medical Imaging Interaction Toolkit (MITK) - a toolkit facilitating the creation of interactive software by extending VTK and ITK," in Medical Imaging 2004, 2004, pp. 16-27.

[23] U. Bland and D. Giavarina, "Lessons in biostatistics," in Biochemia Medica, 2015, vol. 25, no. 2, pp. 141-151. 